\title{
Mitochondrial Reactive Oxygen Species Are Essential for the Development of Psoriatic Inflammation
}

\author{
Soichi Mizuguchi ${ }^{1,2 \dagger}$, Kazuhito Gotoh ${ }^{1 *}$, Yuya Nakashima ${ }^{1}$, Daiki Setoyama ${ }^{1}$, \\ Yurie Takata ${ }^{1}$, Shouichi Ohga ${ }^{2}$ and Dongchon Kang ${ }^{1 *}$ \\ ${ }^{1}$ Department of Clinical Chemistry and Laboratory Medicine, Graduate School of Medical Sciences, Kyushu University, \\ Fukuoka, Japan, ${ }^{2}$ Department of Pediatrics, Graduate School of Medical Sciences, Kyushu University, Fukuoka, Japan
}

\section{OPEN ACCESS}

Edited by:

Vidula Vachharajani, Case Western Reserve University, United States

Reviewed by: Anna Valujskikh,

Cleveland Clinic, United States

Sheikh Fayaz Ahmad, King Saud University, Saudi Arabia

*Correspondence: Kazuhito Gotoh gotou.kazuhito.712@m.kyushuu.ac.jp

Dongchon Kang kang@cclm.med.kyushu-u.ac.jp

${ }^{\dagger}$ These authors have contributed equally to this work

Specialty section:

This article was submitted to Inflammation,

a section of the journal

Frontiers in Immunology

Received: 26 May 2021

Accepted: 16 July 2021

Published: 06 August 2021

Citation:

Mizuguchi S, Gotoh K, Nakashima Y, Setoyama D, Takata Y, Ohga S and Kang D (2021) Mitochondrial

Reactive Oxygen Species Are Essential for the Development of Psoriatic Inflammation.

Front. Immunol. 12:714897. doi: 10.3389/fimmu.2021.714897
Psoriasis is a common immune-mediated, chronic, inflammatory skin disease that affects approximately $2-3 \%$ of the population worldwide. Although there is increasing evidence regarding the essential roles of the interleukin (IL)-23/IL-17 axis and dendritic cell (DC)-T cell crosstalk in the development of skin inflammation, the contributions of mitochondrial function to psoriasis are unclear. In a mouse model of imiquimod (IMQ)-induced psoriasiform skin inflammation, we found that hematopoietic cell-specific genetic deletion of p32/C1qbp, a regulator of mitochondrial protein synthesis and metabolism, protects mice from IMQ-induced psoriatic inflammation. Additionally, we demonstrate that p32/C1qbp is an important regulator of IMQ-induced DC activation, both in vivo and in vitro. We also found that p32/C1qbp-deficient DCs exhibited impaired production of IL$1 \beta, I L-23$, and mitochondrial reactive oxygen species (mtROS) after IMQ stimulation. Because the inhibition of $\mathrm{mtROS}$ suppressed IMQ-induced DC activation and psoriatic inflammation, we presume that $\mathrm{p} 32 / \mathrm{C} 1 \mathrm{qbp}$ and $\mathrm{mtROS}$ can serve as therapeutic targets in psoriasis.

Keywords: C1qbp/p32, dendritic cells, mitochondrial ROS, IL-1 $\beta$, psoriasis

\section{INTRODUCTION}

Psoriasis is a skin disease that affects approximately $2-3 \%$ of the world population, including $10 \%$ of the population in some Northern European countries (1-4). Psoriasis is a common, chronic, relapsing inflammatory dermatitis, which is characterized by epidermal hyperplasia, erythematous plaque formation, and inflammatory cell infiltration in the dermis and epidermis $(5,6)$. Although the complex pathogenesis of psoriasis remains poorly understood, there is increasing evidence that immune function plays pivotal roles in disease development $(5,7,8)$. In the past decade, clinical studies have shown that cytokines released by interleukin-17 (IL-17)-producing T cells (Th17) and dendritic cells (DCs) (e.g., IL-17, tumor necrosis factor- $\alpha$, IL-12/23, and IL-22) are critical for the development of psoriasis $(9,10)$. In addition, multiple biologic agents (including monoclonal antibodies targeting IL-17, tumor necrosis factor- $\alpha$, and IL-12/23) are available for the treatment of moderate to severe psoriasis $(11,12)$. Although biologic agents have robust effects on psoriasis 
treatment, various side effects associated with the administration have become an important problem in recent years (13). Thus, there is a need for novel psoriasis-targeting therapeutic agents.

Psoriasis is a disorder of both innate and adaptive immune systems, in which dendritic cells (DCs), keratinocytes, and T cells have central roles. DCs are key sentinels of the immune system, which bridge the gap between innate and adaptive immunity. Thus far, several studies have indicated that DCs contribute to the initiation of psoriasis through antigen-presenting molecules (e.g., major histocompatibility complex [MHC], MHCII, CD86, and CD40), cytokines (e.g., interferon [IFN], IL-1 $\beta$, IL-23, and IL-6), and chemokine receptors $(6,11,14)$. The proportions of myeloid DCs (mDCs) are increased in psoriasis lesions. In addition, mDCs from psoriasis lesions reportedly activated $\mathrm{T}$ cells to produce both IL-17 and IFN- $\gamma$. These mDCs also regulated the IL-23/IL-17 axis $(15,16)$. Furthermore, DCs activated by Toll-like receptor (TLR) 7-9 stimulation can trigger the onset of autoimmune diseases, including psoriasis and systemic lupus erythematosus (17-20). Although previous studies showed that the TLR7/Th17 axis contributes to psoriatic inflammation (21-23), the detailed molecular mechanism is unknown.

Imiquimod (IMQ) is a TLR 7/8 agonist that has been widely used for the topical treatment of human papilloma virus infection $(17,24,25)$. In humans, IMQ can induce de novo psoriasis lesions and exacerbate preexisting psoriasis lesions $(17,26)$. In addition, daily topical application of IMQ cream on the skin and ears of mice leads to specific dermatitis with many features similar to human psoriasis manifestations $(27,28)$. Because the disease in this mouse model is heavily dependent on the IL-23/IL-17 axis, similar to human psoriasis $(27,28)$, these mice have been widely used for psoriasis-related pathological analysis and drug development. Recent studies have shown that IMQ directly or indirectly affects the mitochondrial electron transport chain (ETC), reactive oxygen species (mtROS), metabolism in vitro $(29,30)$.

The complement component 1q subcomponent binding protein (C1QBP/p32/HABP1) was originally identified as a protein associated with the essential splicing factor ASF/SF2 (31). Our group showed that $\mathrm{p} 32 / \mathrm{C} 1 \mathrm{qbp}$ is processed by proteolytic cleavage of $\mathrm{N}$-terminal amino acids containing the mitochondrial signal sequence; moreover, it is essential for mouse embryonic development dependent on mitochondrial translation $(32,33)$. In macrophages and DCs, we demonstrated that p32/C1qbp is an important innate immunity factor $(34,35)$. Recent reports have shown that $\mathrm{p} 32 / \mathrm{C} 1 \mathrm{qbp}$ mutations impair mitochondrial respiratory chains and cause cardiomyopathy in humans and mice $(36,37)$. Despite the importance of mitochondrial function, the exact mechanisms of $\mathrm{p} 32 / \mathrm{C} 1 \mathrm{qbp}$ in psoriasis remain unclear.

Various genetic, environmental, and immunological factors reportedly contribute to psoriasis development $(8,38,39)$. However, the roles of mitochondria in psoriasis pathogenesis are poorly understood (40). To explore the roles of mitochondria in psoriasis pathogenesis, we employed an IMQ-induced psoriasiform skin disease model. We hypothesized that p32/ Clqbp is involved in immune cell-mediated exacerbation of psoriasis (e.g., via DCs and T cells). Therefore, we developed hematopoietic cell-specific p32/C1qbp-deficient mice. In the present study, we show that $\mathrm{p} 32 / \mathrm{C} 1 \mathrm{qbp}$ is involved in psoriasis pathogenesis through DC activation.

\section{METHODS}

\section{Animals}

C57BL/6 mice were purchased from Japan Clea. Vav1-iCre mice (cat. no. 008610) were obtained from Jackson Laboratory. p32 flox/flox mice have been described previously (33). Age- and sex-matched p32 flox/flox $\mathrm{Vav1-iCre}{ }^{+}(\mathrm{p} 32 \mathrm{cKO})$ and control littermate $\mathrm{p} 32^{\text {flox/flox }} \mathrm{Vav1- \textrm {Cre } ^ { - }}$ (WT) mice were used in this study (41). All mice were maintained on the C57BL/6 background and housed under specific pathogen-free conditions in the animal facility at Kyushu University. The animal research protocols were approved by the Committee of Ethics on Animal Experiments, Faculty of Medical Sciences, Kyushu University.

\section{Cell Cultures}

Bone marrow DCs (BMDCs) were prepared as described elsewhere (42). To generate BMDCs, bone marrow cells were cultured for 6-8 days with GM-CSF (10 ng/mL; PeproTech) in complete medium, comprising RPMI 1640 medium (SigmaAldrich) supplemented with 10\% FBS, penicillin (Thermo Fisher Scientific), streptomycin (Thermo Fisher Scientific), L-glutamine (Thermo Fisher Scientific), non-essential amino acids (Thermo Fisher Scientific), sodium pyruvate (Thermo Fisher Scientific), and 2-mercaptoethanol (Wako Pure Chemical Industries).

\section{In Vivo Treatments}

IMQ-induced psoriasis-like inflammation was elicited and skin severity was assessed as previously described (27). For the IMQinduced psoriasis model, a daily dose of 33-50 mg of IMQcontaining cream, Aldara (Beselna Cream 5\%, Mochida Pharmaceuticals), was applied onto each mouse ear for 4-9 consecutive days. Skin severity was evaluated using the PASI scoring system based on the extent of skin thickness, redness, and scaling. Mitoquinone (MitoQ) is a mitochondria-targeted antioxidant that reduces mtROS (43). C57BL/6 mice were injected intraperitoneally with MitoQ (10 mg/kg, Focus Biomolecules) or mock treatment on days $1,3,6$, and 8 .

\section{Skin Histology}

Skin sections were stained with H\&E. Epidermal thickness was determined by measuring the mean interfollicular distance under the microscope. For IHC staining, skin cryosections were fixed, blocked, and stained with purified rabbit-anti-mouse Ki-67 Ab (1:200 dilution), followed by anti-rabbit IgG secondary antibody (1:200 dilution). Slides were developed with AEC substrate solution (Vector Laboratories) and then counterstained with hematoxylin. Images were acquired at $200 \times$ magnification using a Keyence BZ-X800 microscope. 


\section{Quantitative Real-Time PCR Analyses}

After stimulation or treatment with IMQ $(5 \mu \mathrm{g} / \mathrm{mL})$, BMDCs and mouse ears were collected and washed in PBS, then resuspended in RLT buffer. Total RNA was extracted from BMDCs and mouse ears using an RNeasy Mini Kit (Qiagen), in accordance with the manufacturer's instructions. RNA concentration $(\mathrm{ng} / \mu \mathrm{L})$ and sample purity (260/280 ratio) were measured using the NanoDrop 1000 Spectrophotometer (Thermo Fisher Scientific). Reverse transcription of approximately $500 \mathrm{ng}$ of total RNA was performed with PrimeScript RT reagent Kit (Takara, Japan), in accordance with the manufacturer's protocol. Gene expression was measured by SYBR Green-based qPCR. Relative quantification was performed using the comparative cycle threshold $(\mathrm{Ct})$ method, relative to $18 \mathrm{~S}$ ribosomal RNA. PCR primers are listed in Supplementary Table S1.

\section{FACS}

Mouse ears were collected and split into dorsal and ventral halves. They were then floated for $40 \mathrm{~min}$ at $37^{\circ} \mathrm{C}$ on the surface of $0.5 \%$ trypsin (w/v), which facilitated separation of the dermis from the epidermis. The dermis was cut into small pieces and placed into digestion solution containing collagenase type IV $(1.5 \mathrm{mg} / \mathrm{mL})$. Digestion was performed for $90 \mathrm{~min}$ at $37^{\circ} \mathrm{C}$ with mixing. After the completion of digestion, the solution was mixed thoroughly and filtered through a $70-\mu \mathrm{m}$ nylon filter to obtain a single-cell suspension. For analysis of surface markers, cells were stained in PBS containing $2 \%(\mathrm{v} / \mathrm{v})$ fetal bovine serum with cell surface antibodies. For intracellular staining with antibodies, cells were fixed with $4 \%$ (wt/vol) paraformaldehyde (Wako Pure Chemical Industries) and permeabilized with saponin (0.5\% saponin and $0.5 \%$ BSA in PBS) for $15 \mathrm{~min}$ at room temperature.

BMDCs were stimulated with IMQ ( 5 and $25 \mu \mathrm{g} / \mathrm{mL}$ ) with or without MitoQ (5 and $10 \mu \mathrm{M}$ ). Reagents were added to cells, simultaneously with imiquimod. Antibodies are listed in Supplementary Table S2. Flow cytometry analysis was performed with a FACSVerse using FACSuite software (BD Biosciences).

\section{In Vitro Dermal $\gamma \delta \mathrm{T}$ Cell Stimulation}

Whole skin cell suspensions from the ears of WT or p32cKO mice were cultured in CRPMI in the presence of mouse IL-23 (15 ng/mL, BioLegend), IL-1 $\beta$ (30 ng/mL, PeproTech), or IL-23 and IL-1 $\beta$ for $72 \mathrm{~h}$. Brefeldin A (5 $\mu \mathrm{g} / \mathrm{mL}$, BioLegend) was added $2-3$ $\mathrm{h}$ before intracellular staining and flow cytometry.

Whole skin cell suspensions from the ears of WT or p32cKO mice treated with IMQ for 4 consecutive days were re-stimulated with phorbol myristate acetate (PMA, $1 \mu \mathrm{g} / \mathrm{mL}$, Wako Pure Chemical Industries) and ionomycin $(0.1 \mu \mathrm{g} / \mathrm{mL}$, Wako Pure Chemical Industries) in the presence of brefeldin A ( $5 \mu \mathrm{g} / \mathrm{mL}$, BioLegend) for $2-3 \mathrm{~h}$. They were then stained by the intracellular staining procedure as described to determine IL-17A expression (35).

\section{Mitochondrial ROS Production, Mass, and Membrane Potential}

BMDCs were incubated with $1 \mu \mathrm{M}$ MitoSOX Red mitochondrial superoxide indicator (Thermo Fisher Scientific) at $37^{\circ} \mathrm{C}$ for 15 min in HBSS, washed two times with HBSS, and then analyzed with flow cytometry.
BMDCs were incubated in HBSS with 5 or $25 \mu \mathrm{M}$ IMQ for $10-15 \mathrm{~min}$ at $37^{\circ} \mathrm{C}$. MitoSOX Red (1 $\mu \mathrm{M}$, Invitrogen), MitoTracker Green FM (50 nM, Invitrogen), and TMRM (100 $\mathrm{nM}$, Invitrogen) were respectively added at the beginning of incubation. BMDCs were washed two times with HBSS, then analyzed with flow cytometry.

\section{Metabolism Assays}

BMDCs were analyzed using an XF-24 Extracellular Flux Analyzer (Seahorse Bioscience) (44). Briefly, BMDCs were seeded in XF-24 well culture plates (200,000 cells/well). At the specified time points, BMDCs were washed and analyzed in XF Running Buffer (unbuffered RPMI medium with $10 \mathrm{mM}$ glucose, $10 \%$ fetal calf serum, and $2 \mathrm{mM}$ L-glutamine), in accordance with the manufacturer's instructions, to obtain real-time measurements of the OCR and ECAR. Analyses of the ECAR and/or OCR in response to 5 or $25 \mu \mathrm{g} / \mathrm{mL}$ IMQ were performed.

\section{Immunoblotting Analysis}

For direct immunoblotting, BMDCs were washed in PBS, then lysed with cell lysis buffer (Cell Signaling Technology). The lysate was eluted with sample buffer and boiled for $5 \mathrm{~min}$ at $97^{\circ} \mathrm{C}$. SDS-PAGE was performed with $8 \%-15 \%$ polyacrylamide gels using a constant current of $20 \mathrm{~mA}$. The gels were transferred to Immobilon-P membranes (Merck Millipore) using a semidry blotting device for $15 \mathrm{~min}$, with a constant current of $2.5 \mathrm{~A}$. The membranes were blocked with Blocking One (Nacalai Tesque) and incubated with the appropriate primary antibody overnight at $4^{\circ} \mathrm{C}$ in Can Get Signal Solution 1 (Toyobo Co., Ltd.) (Supplementary Table S2). The membranes were subsequently incubated with the appropriate secondary antibody for 1 hour at RT in Can Get Signal Solution 2 (Toyobo Co., Ltd.), then washed again. Signals were visualized by chemiluminescence using a Clarity ECL Substrate (BIO-RAD) and an ImageQuant LAS4000 Mini image analyzer (GE Healthcare), in accordance with the manufacturer's protocols.

\section{ELISA}

For cytokine quantification of cell-free supernatants, ELISA kits for murine IL-1 $\beta$ and IL-23 from BioLegend were used as directed by the manufacturer. Data are expressed as the means \pm SDs of triplicate wells.

\section{Quantification and Statistical Analysis}

Statistical analyses were performed using GraphPad Prism. All experiments were performed with at least two independent biological replicates. The results are expressed as the means \pm SDs or means \pm SEMs of the independent experiments. P-values were calculated using two-tailed Student's t-tests.

\section{RESULTS}

\section{Absence of p32/C1qbp in Hematopoietic Cells Protects Mice From IMQ-Induced Psoriatic Inflammation}

We found that the gene expression of p32/C1qbp was significantly increased in lesional skin from patients with 
psoriasis, compared with healthy controls (38) (Figure 1A). Thus, we investigated the relationship between $\mathrm{p} 32 / \mathrm{C} 1 \mathrm{qbp}$ and IMQ-induced psoriatic inflammation using a mouse model. The gene expression of $\mathrm{p} 32 / \mathrm{C} 1 \mathrm{qbp}$ was increased during IMQinduced psoriatic inflammation, similar to human psoriasis (Supplementary Figure 1A). To evaluate the role of immunological $\mathrm{p} 32 / \mathrm{C} 1 \mathrm{qbp}$ in psoriatic inflammation, we generated a hematopoietic-specific p32/C1qbp conditional knockout (cKO) mouse strain by mating loxP-flanked p32/ C1qbp gene mutation $\mathrm{p} 32^{\text {flox/flox }}$ mice with Vav1-Cre transgenic mice. Repetitive topical application of IMQ to the ears of WT mice (Vav1-Cre ${ }^{-}$p32 flox/flox mice) induced psoriatic inflammation with marked erythema, scaling, and stiffness (Figures 1B-D). Notably, p32cKO mice had reduced the severity of psoriatic symptoms, compared with WT mice (Figures 1B-D). Histological examination also revealed that IMQ-treated skin in WT mice was characterized by epidermal hyperplasia, with massive infiltration of inflammatory cells (Figures 1E, F and Supplementary Figure 1B). In contrast, p32cKO mice did not exhibit these histological changes following IMQ treatment (Figures 1E, F). The increased ear thickness following IMQ application in WT mice was abrogated in p32cKO mice (Figure 1F). Additionally, the proportion of epidermal keratinocytes expressing the proliferation marker Ki67 was smaller in skin from IMQ-treated p32cKO mice, compared with skin from IMQ-treated WT mice (Figure 1G). These results demonstrate that $\mathrm{p} 32 / \mathrm{C} 1 \mathrm{qbp}$ in hematopoietic cells is essential for the development of IMQ-induced psoriatic dermatitis.

\section{p32/C1qbp Regulates IMQ-Induced Psoriatic Inflammation}

Because the IL-23/IL-17 axis is an important pathway in psoriasis pathogenesis, we examined whether the loss of p32/ C1qbp in hematopoietic cells inhibit the IL-23/IL-17-mediated pathogenesis of psoriasis-like dermatitis. Quantitative RT-PCR analysis revealed significantly reduced expression levels of typical psoriasis lesion-associated antimicrobial peptides (S100a8) in ears from p32cKO mice (Figure 2A). In addition, mRNA expression levels of proinflammatory cytokines (e.g., IL-17, IL22 , IL-23, IL-6, and IL-1 $\beta$ were significantly reduced in skin from IMQ-treated p32cKO mice (Figures 2B, C). Therefore, we concluded that p32/C1qbp in immune cells is a key driver in the development of IMQ-induced psoriatic dermatitis.

Psoriasis is a disorder of both innate and adaptive immune systems involving DCs, T cells, and neutrophils have central roles. Indeed, we found that neutrophils predominantly infiltrated into the ears from IMQ-treated WT mice (Figure 2D and Supplementary Figure 2A). In contrast, neutrophil infiltration was significantly reduced in the skin from $\mathrm{p} 32 \mathrm{cKO}$ mice. Because the loss of p32/C1qbp did not affect neutrophil migration (Supplementary Figure 2B), the reduced neutrophil infiltration associated with $\mathrm{p} 32 / \mathrm{C} 1 \mathrm{qbp}$ deficiency may be dependent on other cells (e.g., DCs and $\gamma \delta$ T cells). We also found that the proportion of IL-17-producing $\gamma \delta$ T cells was decreased in the ears from IMQtreated p32cKO mice (Figure 2E, middle lane, Supplementary Figure 2C). Because the induction of IL-17A by phorbol myristate acetate (PMA) + ionomycin stimulation occurred normally in $\gamma \delta$ (Figure 2E, right lane) and $\alpha \beta$ T cells (Supplementary Figure 2D) from $\mathrm{p} 32 \mathrm{cKO}$ mice in vitro, we presumed that $\mathrm{p} 32 / \mathrm{Clqbp}$ acts independently of a protein kinase $\mathrm{C}$ and $\mathrm{Ca}^{2+}$ signaling pathway in $\mathrm{T}$ cells. DCs became activated and promoted inflammatory responses after IMQ application. Indeed, we found that DCs from the ears of WT mice were activated after IMQ stimulation (Figure 2F, Supplementary Figure 2E). In contrast, DCs from the ears of p32cKO mice demonstrated impaired activation of CD40 and CD86 following IMQ stimulation (Figure 2F). To investigate whether DCs are directly involved in the exacerbation of psoriasis, we subcutaneously administered IMQ-treated WT or p32deficient DCs to wild-type mice. At 48 hours after transfer, redness was significantly exacerbated in ears with IMQ-treated WT BMDCs (Supplementary Figure 2F), compared with IMQtreated p32 deficient DCs. Therefore, we concluded that p32/ C1qbp regulates the exacerbation of psoriatic inflammation through DC activation, rather than $\mathrm{T}$ cell activation and neutrophil migration.

\section{p32/C1qbp Is a Key Regulator of IMQ- Induced DC Activation In Vitro}

Because we thought that p32/C1qbp-dependent DC activation was associated with the exacerbation of psoriasis, we analyzed the relationship between $\mathrm{p} 32 / \mathrm{C} 1 \mathrm{qbp}$ and DC activation following IMQ stimulation in vitro. DCs express TLR7 and produce both proinflammatory cytokines and type I interferon (IFN) in an NF$\kappa \mathrm{B}$ or interferon regulatory factor (IRF)-dependent manner, following IMQ stimulation. Consistent with our in vivo data (Figure 2C), we found that IMQ-induced transcription and production of both IL-1 $\beta$ and IL-23 were impaired in BMDCs from $\mathrm{p} 32 \mathrm{cKO}$ mice (p32 $2^{-/-} \mathrm{DCs}$ ) (Figures 3A-D). In addition, the loss of $\mathrm{p} 32 / \mathrm{C} 1 \mathrm{qbp}$ suppressed the expression of Il-12b and Ifnb1 following IMQ stimulation (Figure 3A). Because phosphorylation levels of $I K K \alpha / \beta$, I $\mathrm{B}$, and $N F-\kappa B$ were suppressed in $\mathrm{p}^{2} 2^{-/-}$DCs stimulated with IMQ (Figure 3E and Supplementary Figure 3A), we presumed that $\mathrm{p} 32^{-/-}$DCs exhibit reduced the production of proinflammatory cytokines. IL-1 $\beta$ and IL-23 induced IL-17 production from $\gamma \delta$ T cells. Indeed, we found that $\gamma \delta \mathrm{T}$ cells from the ears of WT mice enhanced IL-17 production by stimulating IL-1 $\beta$ and IL-23 in vitro (Supplementary Figure 3B most right lane). Because IL$1 \beta$-induced IL-17 production by $\gamma \delta \mathrm{T}$ cell was unaffected by $\mathrm{p} 32 /$ C1qbp gene deficiency and IL-23 did not induce IL-17 production in $\gamma \delta \mathrm{T}$ cells (Supplementary Figure 3B, middle two lane), we speculated that psoriatic inflammation could be alleviated by reducing IL- $1 \beta$ production from $\mathrm{p} 32^{-/-} \mathrm{DCs}$ in vivo.

Although cytokines such as IL-17, IL-23, and IL-1 $\beta$ are mainly involved in the exacerbation of psoriasis, DC-T cell crosstalk through CD80/CD86 also affects psoriasis. We showed that $\mathrm{p} 32 / \mathrm{C} 1 \mathrm{qbp}$ was involved in DC maturation following IMQ administration in vivo (Figure $2 \mathbf{F}$ ). The loss of p32/C1qbp in BMDCs led to suppression of DC maturation by IMQ stimulation in vitro (Figure $3 \mathbf{F}$ and Supplementary Figures 3C, D). These findings suggest that p32/C1qbp is associated with psoriasis pathology through DC activation. 


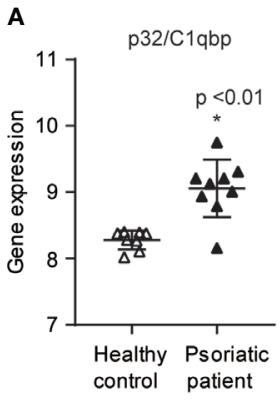

B

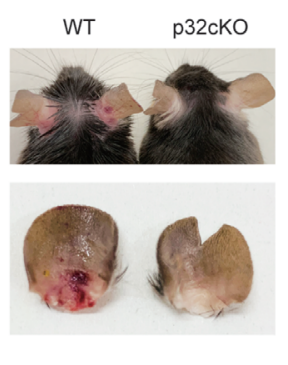

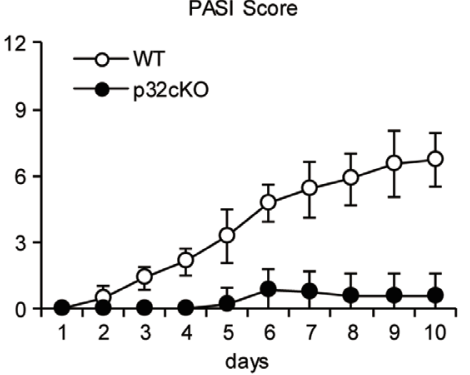

D Score of redness

Score of scaling

Score of stiffness
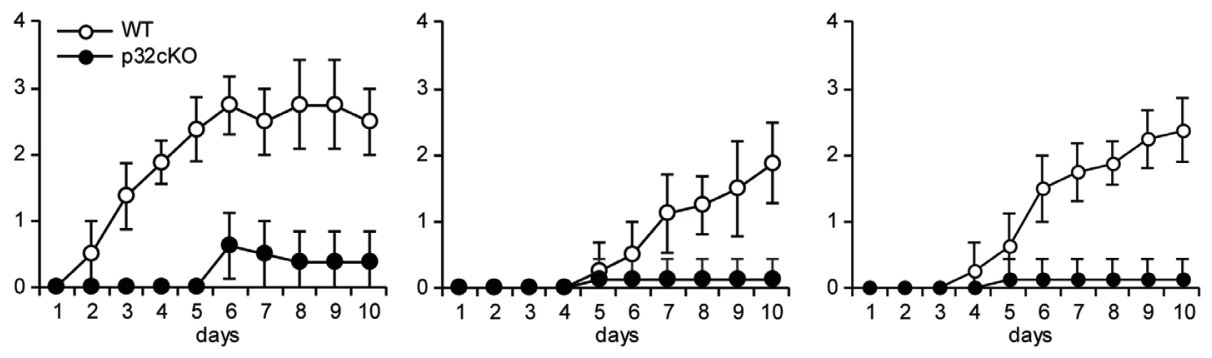

E

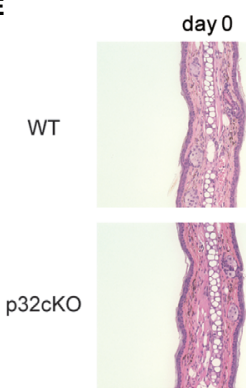

day 5

day 10
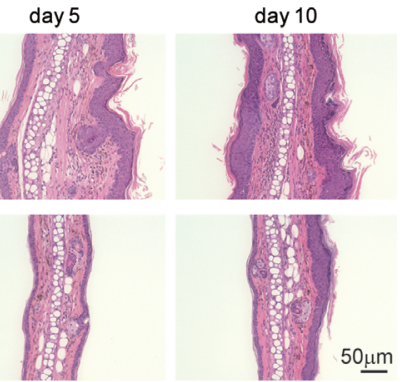

F

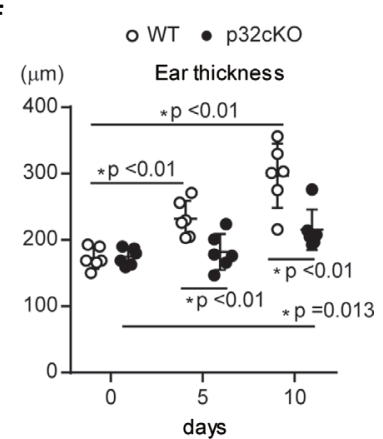

G
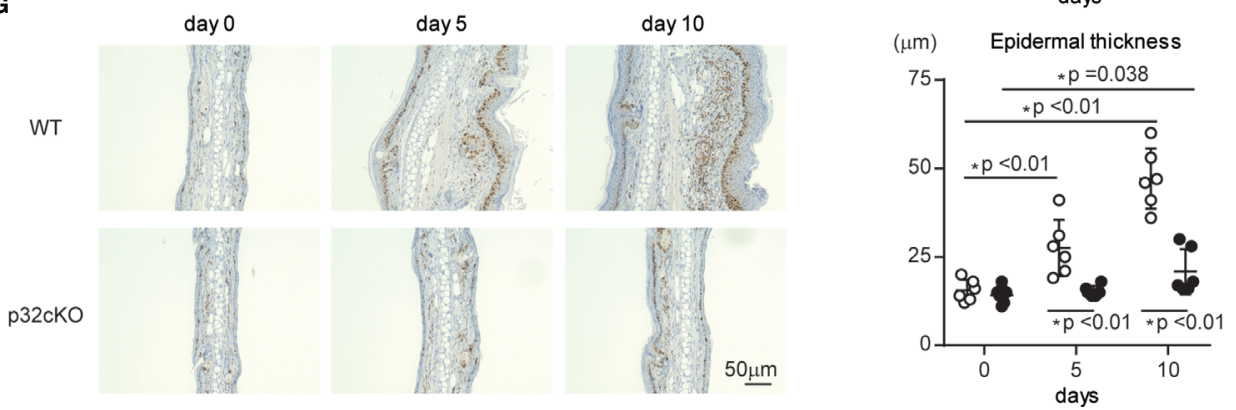

FIGURE 1 | Effects of p32/C1qbp deficiency on psoriatic inflammation. (A) Gene expression patterns of p32/C1qbp in lesions from patients with psoriasis ( $\mathbf{n}$ = 9) versus healthy individuals ( $\mathrm{n}=8$ ) from Gene Expression Omnibus (http://www.ncbi.nlm.nih.gov/geo; accession number GSE75890). (B-D) WT and p32cKO mice were topically treated with IMQ for 9 consecutive days. Representative gross appearances of ears from WT and p32cKO mice that were treated daily with topical IMQ (B). Cumulative Psoriasis Area Severity Index (PASI) scores (C) were calculated based on the individual scores for redness, scaling, and stiffness (D) daily until 9 days after IMQ application. Data are shown as means \pm SDs $(n=8)$. (E-G) Microscope images of cross-sections of mouse ears stained with hematoxylin and eosin (E) and subjected to immunohistochemical analysis of Ki-67 (G). Changes in ear (F upper) and epidermal (F lower) thickness over time. Scale bars, 50 um. Data are shown as means \pm SDs (A, C, D, F). ${ }^{*} p<0.05$ versus WT mice. Data are representative of at least three (A-G) independent experiments.

\section{p32/C1qbp Supports Mitochondrial ROS Generation After IMQ Stimulation}

Because we found that $32 / \mathrm{C} 1 \mathrm{qbp}$ is involved in IMQ-induced DC activation in vitro (Figure 3), we investigated the underlying molecular and metabolic mechanisms. IMQ stimulation induces glycolytic and mitochondrial metabolic changes in DCs. Thus, we examined whether IMQ influenced glycolysis and mitochondrial oxygen consumption using a flux analyzer assay. We measured the 

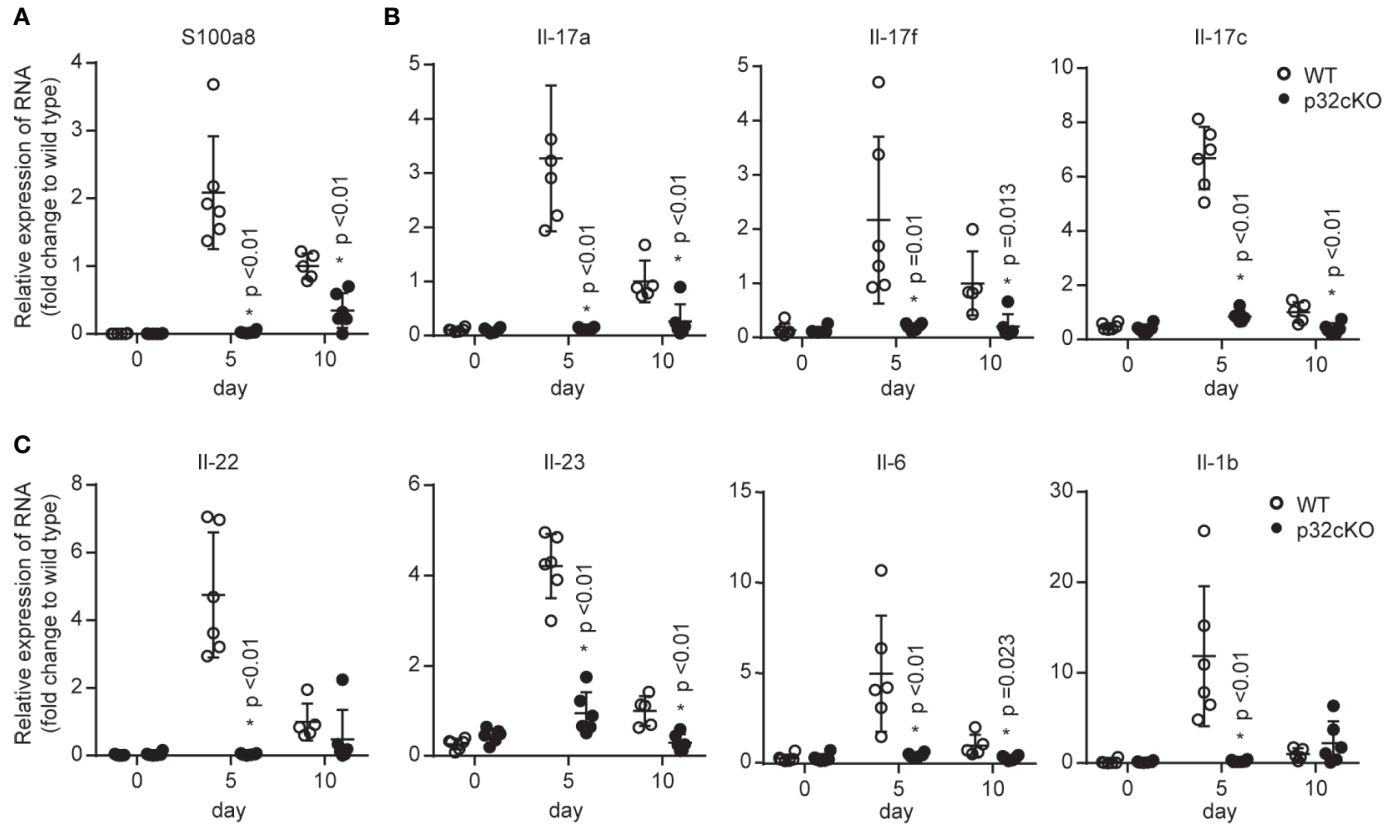

D

Neutrophils

E

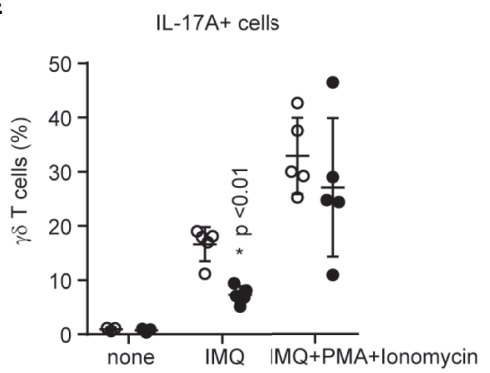

F

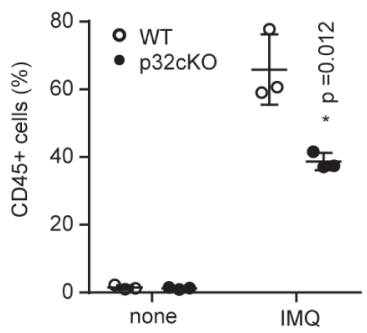

CD40

CD86
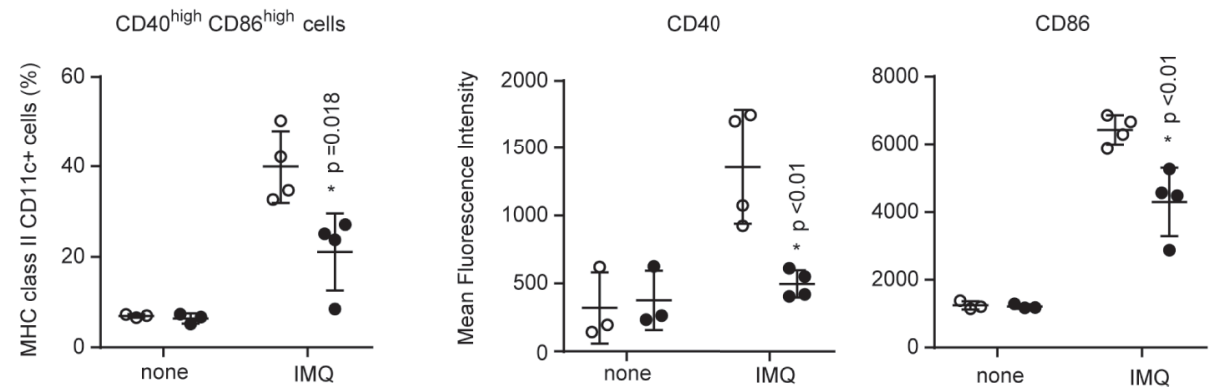

FIGURE 2 | Roles of p32/C1 qbp in psoriatic inflammation in vivo. (A-C) WT and p32cKO mice were treated with IMQ for 9 consecutive days. Real-time PCR analysis was performed to assess mRNA levels in IMQ-treated mouse ears on days 5 and $10(n \geq 3)$. The results were normalized to $18 S$ expression and are shown as means \pm SDs. (D-F) WT and p32cKO mice $(n \geq 3)$ were treated with IMQ for 4 consecutive days. Infiltration of neutrophils (D), IL-17 ${ }^{+} \gamma \delta$ T cells $(\mathbf{E})$, and $\mathrm{CD} 40^{+}$ $\mathrm{CD}^{+} 6^{+} \mathrm{DCs}$ populations in the ears were analyzed by flow cytometry. IL-17 ${ }^{+} \gamma \delta \mathrm{T}$ cell numbers were analyzed before (E, left and middle lanes) and after (E, right lane) PMAVionomycin (PI) stimulation in vitro. Expression levels of CD40 and CD86 were measured in DCs obtained from the ears of WT and p32 cKO mice. Results are expressed as means \pm SDs. Data are shown as means \pm SDs $(\mathbf{A}-\mathbf{F})$. ${ }^{*} \mathrm{p}<0.05$ versus $\mathrm{WT}$ mice. Data are representative of at least three (A-F) independent experiments.

extracellular acidification rate (ECAR) as an index of lactate production and glycolysis in BMDCs; we also measured the oxygen consumption rate (OCR) as an indicator of mitochondrial respiration in BMDCs. Indeed, we found that the
ECAR rapidly increased in WT DCs that had been subjected to IMQ stimulation (Figure 4A). In contrast, the change in a glycolytic flux by IMQ stimulation was negligible in $\mathrm{p} 32^{-/-}$DCs (Figure 4B). We also found that the OCR rapidly decreased in WT 

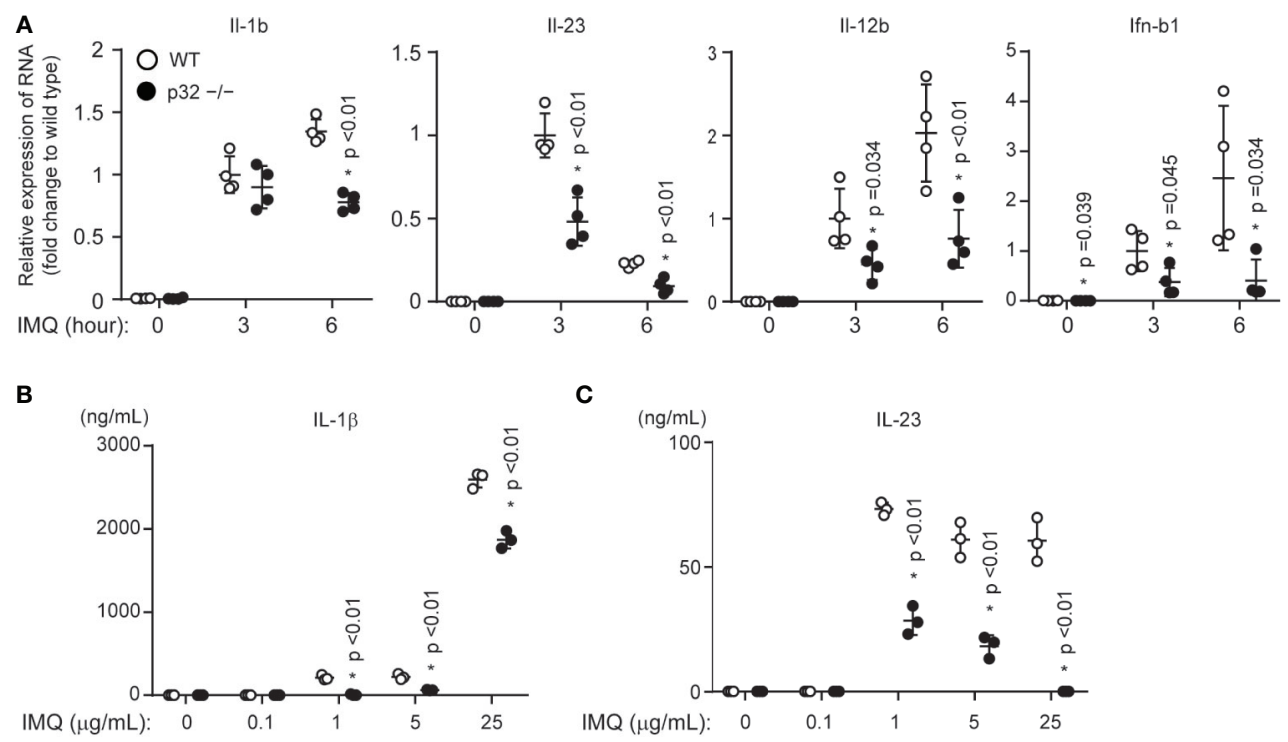

C

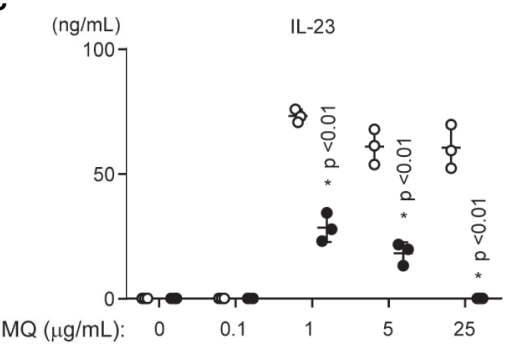

D

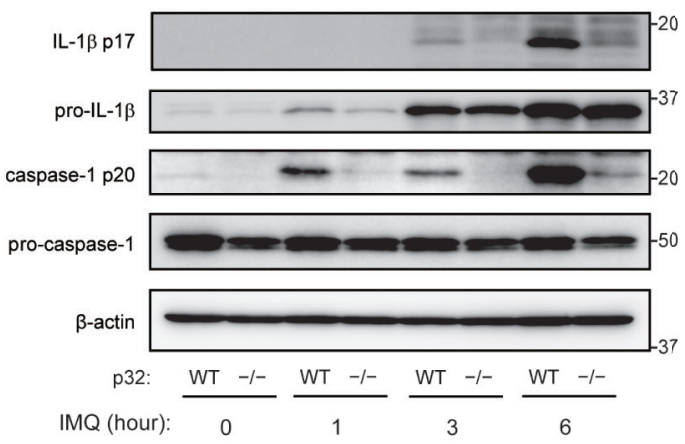

E

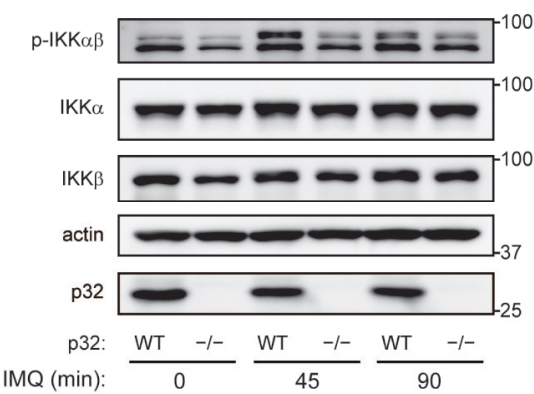

$\mathbf{F}$

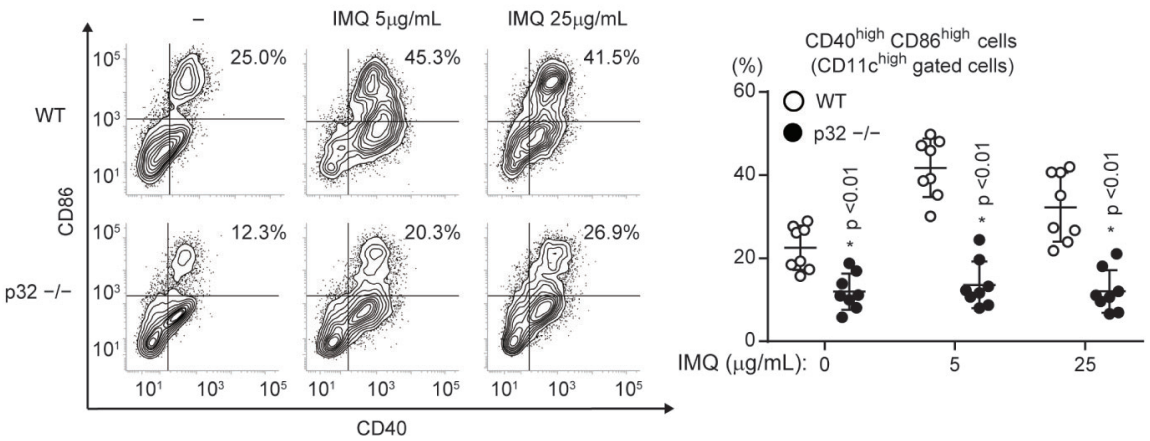

FIGURE 3 | Roles of p32/C1qbp in vitro. (A) Real-time PCR analyses of II-1b, II-23, II-12b, and Ifnb1 expression levels in WT and p32 ${ }^{-/-}$DCs stimulated with 5 $\mu$ g/ $\mathrm{mL} I \mathrm{IMQ}$ for the indicated intervals. Data are shown as means \pm SEMs after normalization to expression of the gene encoding $18 \mathrm{~S}$ ribosomal RNA (18S rRNA). $(\mathbf{B}, \mathbf{C})$ Levels of IL-1 $\beta$ (B) and IL-23 (C) in cell culture supernatants were compared between WT and p32 $2^{-/-} \mathrm{BMDCs}\left(2 \times 10^{5}\right.$ cells/well) after $24 \mathrm{~h}$ of stimulation with IMQ. Data are shown as means \pm SDs of triplicate wells. (D, E) WT and p32 ${ }^{-/-}$DCs were stimulated with $5 \mu \mathrm{g} / \mathrm{mL} \mathrm{IMQ}$ for the indicated intervals and analyzed for IL-1 $\beta$ p 17 , caspase-1 p20 (D), and phosphorylation of IKK- $\alpha \beta$ (E). $\beta$-Actin was used as an internal control. (F) Expression levels of cell surface markers of CD86 and CD40 in WT (upper) and p32 $2^{-/-}$(lower) BMDCs left untreated (-) or stimulated for $12 \mathrm{~h}$ with IMQ and analyzed using flow cytometry to quantify cell surface staining of CD86 and CD40. Numbers in top right corners indicate the percentages of $\mathrm{CD}_{6} 6^{+} \mathrm{CD} 40^{+}$cells. Data are representative of two (A) and three (B-F) independent experiments.

DCs that had been subjected to IMQ stimulation (Figure 4C). In contrast, the OCR in $\mathrm{p} 32^{-/-}$DCs was severely suppressed due to the inhibition of mitochondrial translation and resultant mitochondrial respiratory chain complex I deficiency, and therefore was not changed by IMQ injection (Figure 4D). While the mitochondrial mass of WT DCs was increased by IMQ, the mass in $\mathrm{p} 32^{-/-}$DCs originally increased and was little affected by IMQ (Supplementary Figure 4A). The mitochondrial membrane 

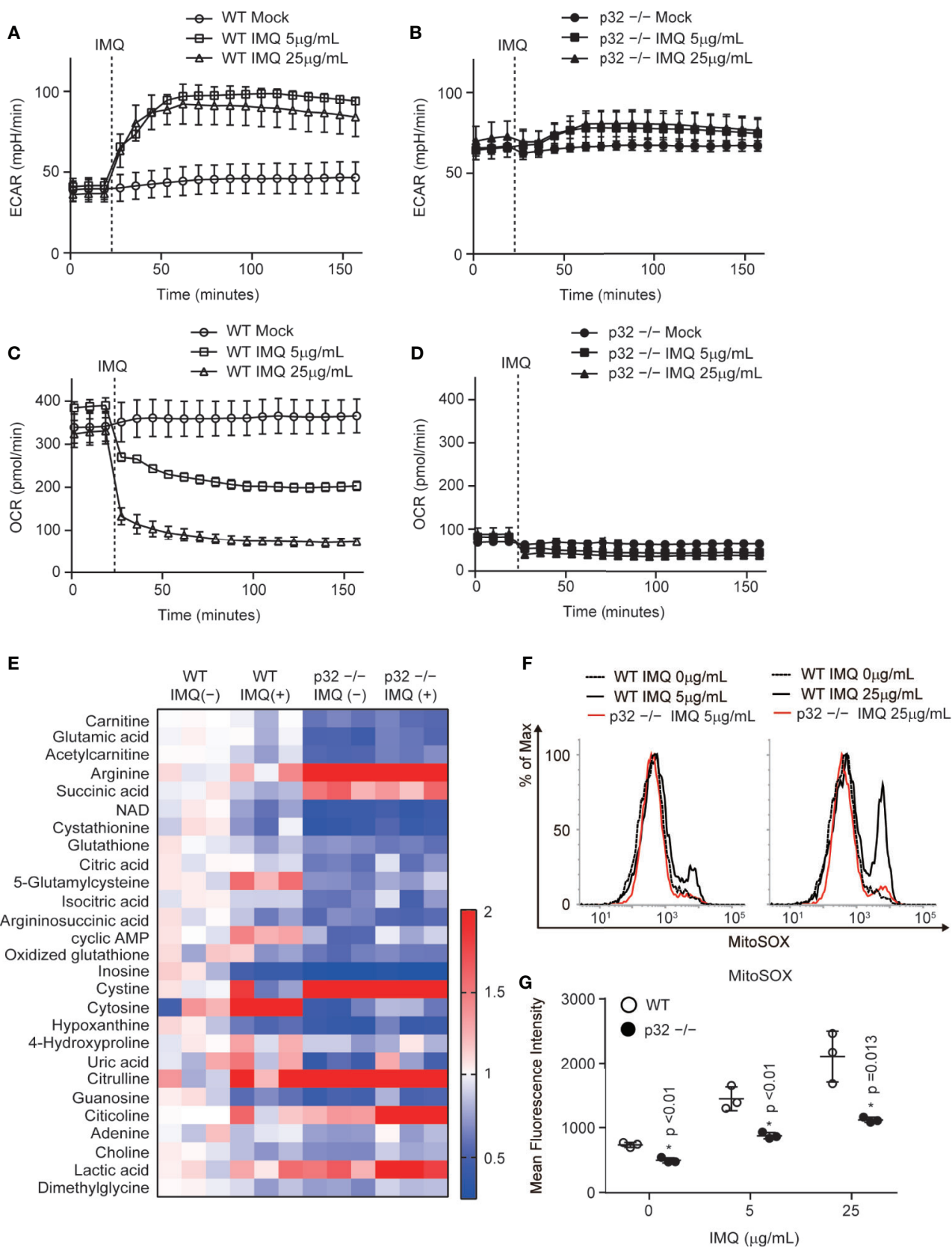

FIGURE 4 | p32/C1 qbp regulates mitochondrial metabolism and ROS after IMQ stimulation. (A-D) Real-time changes in the ECAR (A, B) or OCR (C, D) in WT (A, C) and p32 $2^{-/-}$(B, D) DCs treated with $5 \mu \mathrm{g} / \mathrm{mL} I M Q$. Vertical dotted lines indicate the initiation of treatment. Data are shown as means \pm SDs of triplicate wells. (E) Comparisons of the amounts of metabolites between WT and p32 ${ }^{-/-}$BMDCs with or without IMQ stimulation. Heat map of WT and p32 ${ }^{-/-}$DC metabolites that exhibited statistically significant changes (P < 0.05). (F, G) Flow cytometry histograms (F) and quantification (G) of the expression of mitochondrial ROS (MitoSOX) in WT and p32 $2^{-/}$DCs treated with 5 or $25 \mu \mathrm{g} / \mathrm{mL} I M Q$. Data are shown as means \pm SDs of triplicate wells. Data are representative of two (E) and three (A-D, F-G) independent experiments.

potential was similarly decreased after IMQ stimulation both in WT and $\mathrm{p} 32^{-/-}$DCs. (Supplementary Figure 4B). To explore the detailed metabolic mechanism by which p32 controls IMQdependent DC activation, we compared intracellular metabolites between WT and p32 $2^{-/-}$DCs. Indeed, metabolites of the fatty acid oxidation (carnitine and acetylcarnitine) and tricarboxylic acid
(TCA) cycle (citric acid and isocitric acid) were significantly reduced in $\mathrm{p} 32^{-/-}$DCs before IMQ stimulation (Figure $4 \mathrm{E}$ and Supplementary Figures 4C, D). Furthermore, the metabolites of glutathione metabolism (glutamic acid, glutathione, 5-glutamylcysteine, and oxidized glutathione) were significantly lower in $\mathrm{p} 32^{-/-}$DCs (Figure 4E). Because the concentration of 
oxidized glutathione was decreased in $\mathrm{p} 32^{-1-}$ DCs, we expected that the generation of mtROS would be decreased in $\mathrm{p} 32^{-/-}$DCs.

IMQ-induced inflammasome activation requires mtROS through mitochondrial respiratory chain complex I activity. In addition, IMQ induces the production of IL- $1 \beta$ through mtROSand caspase-1-dependent NLRP3 inflammasome activation. Indeed, we found that the inhibition of mitochondrial respiratory chain complex I could suppress a burst of mitochondrial ROS by IMQ stimulation, as well as the production of IL-1 $\beta$ and IL-23 in WT DCs (Supplementary Figures 5A-C). Because IMQ stimulation induces a burst of mitochondrial ROS, we compared IMQ-induced mtROS between WT and $\mathrm{p} 32^{-/-}$DCs. We found that the level of IMQinduced mtROS was decreased in $\mathrm{p} 32^{-/-}$DCs (Figures 4F, G). In addition, IMQ-induced caspase-1 activation was decreased in p $32^{-/-}$DCs (Figure 3D). These findings indicate that p32/C1qbp supports IMQ-induced IL-1 $\beta$ through mtROS in vitro.

\section{Mitochondrial ROS Promote IMQ-Induced DC Activation In Vitro}

We showed that $\mathrm{p} 32 / \mathrm{C} 1 \mathrm{qbp}$ supports IMQ-induced DC activation (Figure 3). In addition, the loss of p32/C1qbp suppressed the generation of mtROS after IMQ stimulation (Figure 4). Thus, we presumed that mtROS could influence DC activation by IMQ stimulation. Indeed, we found that MitoQ, a mitochondria-targeted antioxidant that reduces mtROS, could suppress IMQ-induced mtROS (Figure 5A). In addition, MitoQ inhibited IMQ-induced surface expression of both CD40 and CD86 in WT DCs (Figures 5B, C). Although five $\mu \mathrm{M}$ MitoQ was not sufficient to suppress the production of IL-1 $\beta$ and IL-23, treatment with ten $\mu \mathrm{M}$ MitoQ significantly suppressed the production of IL-1 $\beta$ and IL-23 after IMQ stimulation (Figures 5D, E). These results indicate that the inhibition of mtROS suppresses IMQ-induced DC activation in vitro.

\section{Inhibition of Mitochondrial ROS Suppresses IMQ-Induced Psoriatic Inflammation}

Our data indicated that $\mathrm{p} 32 / \mathrm{C} 1 \mathrm{qbp}$ supports IMQ-induced DC activation through mtROS in vitro (Figures 4 and 5). To examine the role of mtROS in IMQ-induced psoriatic inflammation, we analyzed psoriatic symptoms in ears that had been subjected to repetitive topical application of IMQ after intraperitoneal injection of MitoQ $(10 \mathrm{mg} / \mathrm{kg})$ in WT mice. We found that psoriatic symptom severity was reduced in mice treated with MitoQ, compared with WT mice that had received mock treatment (Figure 6A). Histological examination also revealed that IMQ-treated skin from WT mice with MitoQ exhibited reduced epidermal hyperplasia with massive infiltration of inflammatory cells (Figures 6B, C). Consistent with our in vitro data (Figures 5D, E), the expression levels of Il-1b and Il-23 in the ears of WT mice treated with MitoQ were significantly decreased on day 5 after IMQ treatment (Figure 6D). Although we could not determine whether MitoQ indeed suppressed mtROS in DCs, the inhibition of mtROS was sufficient to suppress IMQ-induced psoriatic inflammation.

\section{DISCUSSION}

p32/C1qbp is a gene associated with psoriasis in humans (Figure 1A). Our mouse genetics study revealed the contribution of a p32/C1qbp-dependent mechanism to the exacerbation of psoriasis. We also showed that mtROS-dependent IL- $1 \beta$ is involved in the exacerbation of psoriatic inflammation. The inhibition of mtROS was effective for reducing disease severity in our mouse model of psoriasis (Figure 6). Our findings suggest that p32/C1qbp, which functions as a multifunctional chaperone protein in mitochondria, is an important genetic factor involved in psoriasis.

Previous studies showed that DC activation by TLR agonists contributes to the exacerbation of several diseases (e.g., autoimmune diseases, inflammatory diseases, allergies, and cancer) $(20,45-47)$. In particular, DCs activated by TLR agonists reportedly induce Th17 differentiation and exacerbate autoimmune diseases (e.g., psoriasis and experimental autoimmune encephalomyelitis) $(48,49)$. The mechanism by which IMQ-treated DCs induce Th17 differentiation in psoriatic inflammation has been clarified by recent studies $(22,30,50)$. Although our study does not fully show the mechanism underlying the exacerbation of psoriasis through IMQ-induced DC activation, we have elucidated a portion of the mechanism underlying exacerbation of psoriasis by $\mathrm{p} 32 / \mathrm{C} 1 \mathrm{qbp}$ and $\mathrm{mtROS}$ dependent pathways. To clarify the relationship between p32/ C1qbp and psoriatic inflammation, it will be necessary to cross CD11c-cre and p32 flox mice and analyze them using a psoriatic model.

Previous studies showed that IL-1 $\beta$ is involved in the pathogenesis of psoriasis and psoriatic arthritis $(5,39,51,52)$. Although the inhibition of IL-1 $\beta$ is not fully established as an effective treatment for psoriasis, treatment with IL- $1 \beta$ inhibitors reportedly improved disease in several patients with psoriasis $(53,54)$. Because IL- $1 \beta$ has a critical role in the differentiation and activation of IL-17-producing T cells (55-57), IL-1 $\beta$ may be directly or indirectly involved in the exacerbation of psoriasis by promoting Th17 cell polarization. A previous study showed that DCs in IMQ-treated skin mainly produce IL-1 $\beta$ (52); the study also showed that the IL-1 $\beta$-IL-1R signaling pathway contributes to skin inflammation and psoriasis pathogenesis. Despite differences in major IL- $1 \beta$-producing cells between mouse models and human disease, we speculate that the inhibition of IL- $1 \beta$ production can be a treatment for psoriasis, similar to the inhibition of IL-17 production.

ROS are reportedly pathogenic in psoriasis (58-60). In addition, IMQ-induced psoriatic dermatitis can be prevented by ROS (61). Although there are many sources of ROS (e.g., NADPH oxidases [NOX], mitochondrial ETC, xanthine oxidase, uncoupled endothelial nitric oxide synthase [eNOS], cytochrome P-450 oxygenase, and cyclooxygenase), there remains a lack of clarity regarding the specific ROS pathway involved in the exacerbation of psoriasis $(60,62)$. In this study, we determined 

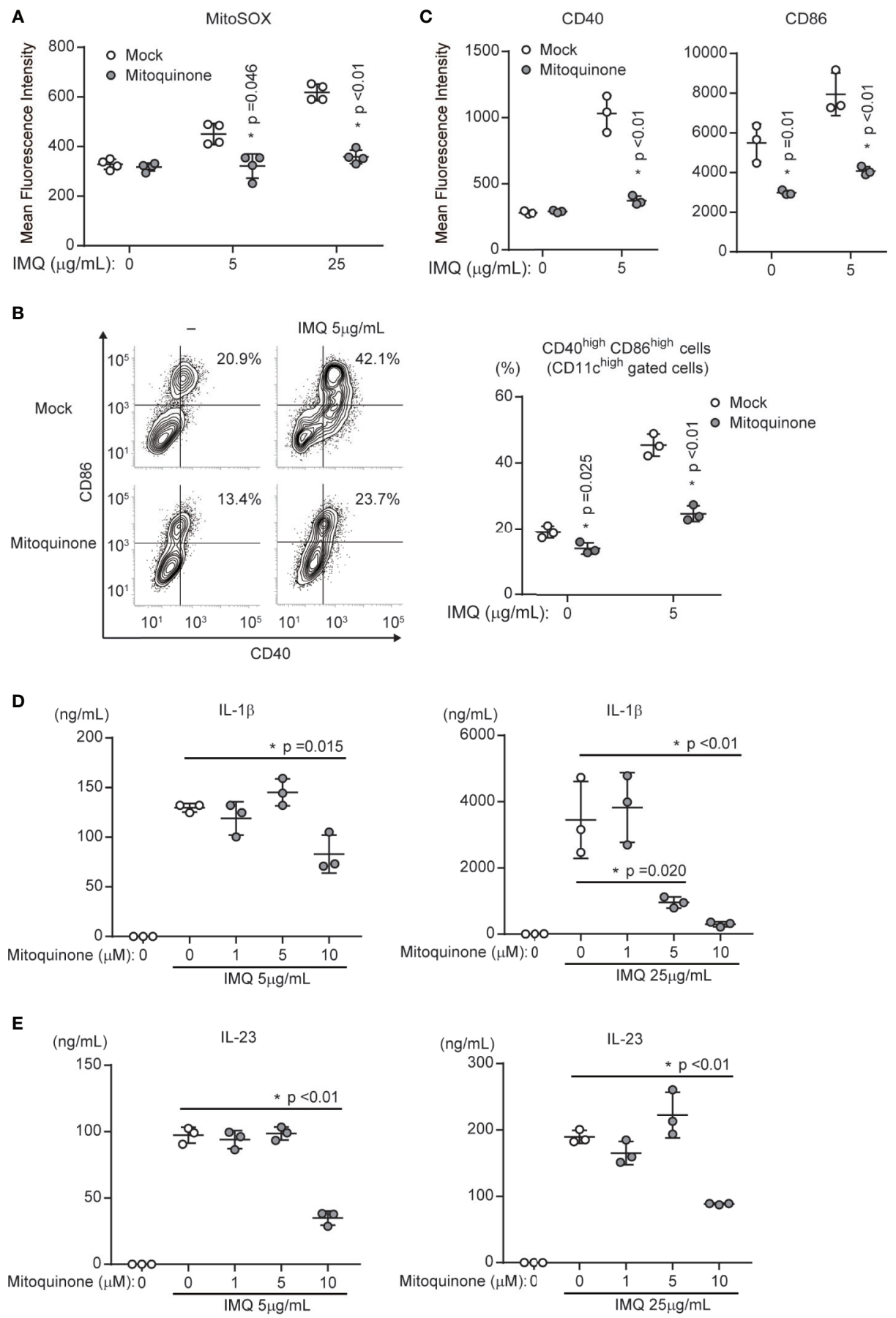

FIGURE 5 | Mitochondrial ROS are required for DC activation by IMQ stimulation. (A) WT DCs in the presence or absence of mitoquinone were stimulated with 5 or $25 \mu \mathrm{g} / \mathrm{mL} \mathrm{IMQ}$ and analyzed for the expression of mitochondrial ROS (MitoSOX). Data are shown as means \pm SDs $(n=4)$. (B, C) WT DCs in the presence or absence of mitoquinone were stimulated with IMQ and analyzed to quantify the expression of cell surface markers CD86 and CD40 (C). Numbers in the top right corners indicate the percentages of $\mathrm{CD}_{8} 6^{+} \mathrm{CD} 40^{+}$cells (B, left). Pooled results from three independent experiments are shown (B, right). Data are shown as means \pm SDs of triplicate samples. (D, E) Cytokine production was compared among WT DCs exposed to mock treatment or mitoquinone. Cells were stimulated with 5 or $25 \mu \mathrm{g} / \mathrm{mL}$ IMQ for $24 \mathrm{~h}$ in the presence or absence of inhibitors. Data indicate the levels of IL-1 $\beta$ (D) and IL-23 (E) in cell culture supernatants (means \pm SDs of triplicate wells). Data are shown as means \pm SDs. ${ }^{*} p<0.05$ versus mock treatment. Data are representative of three independent experiments. 

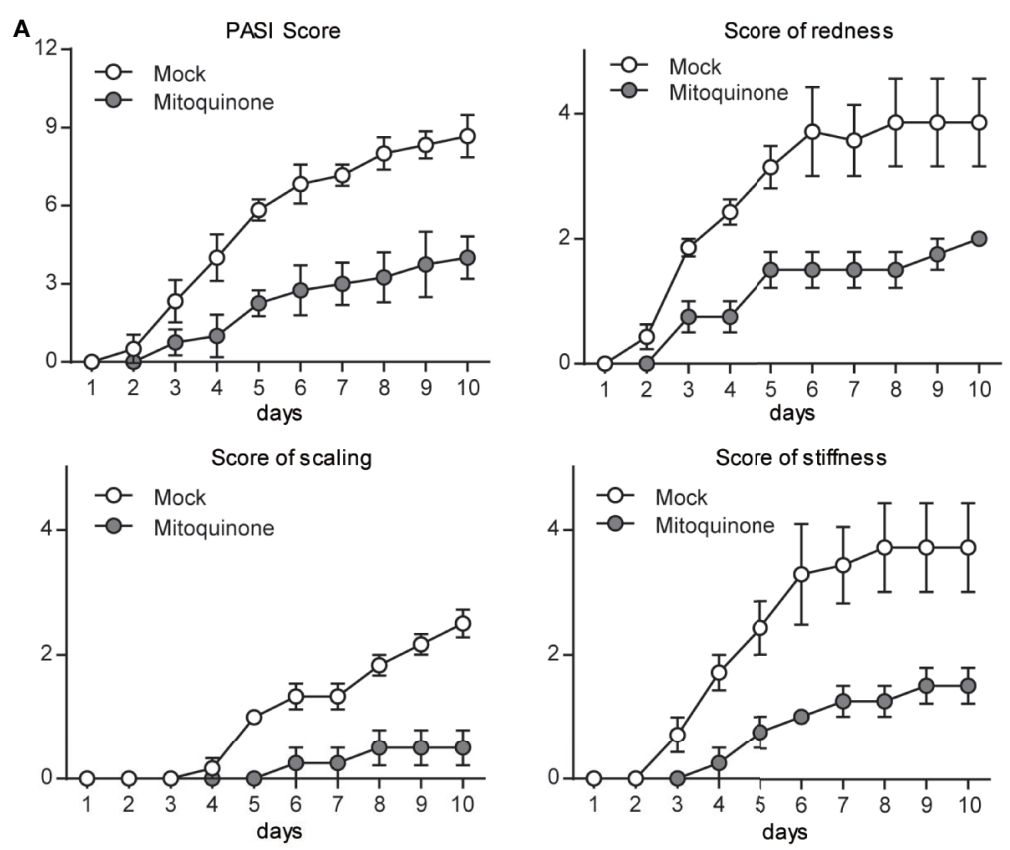

B
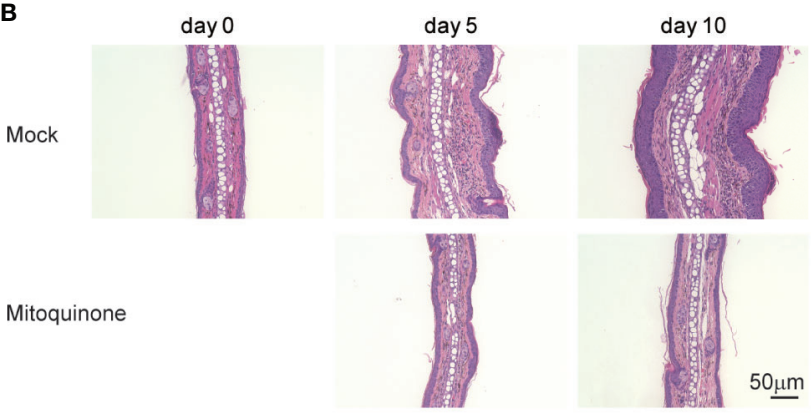

C Mitoquinone
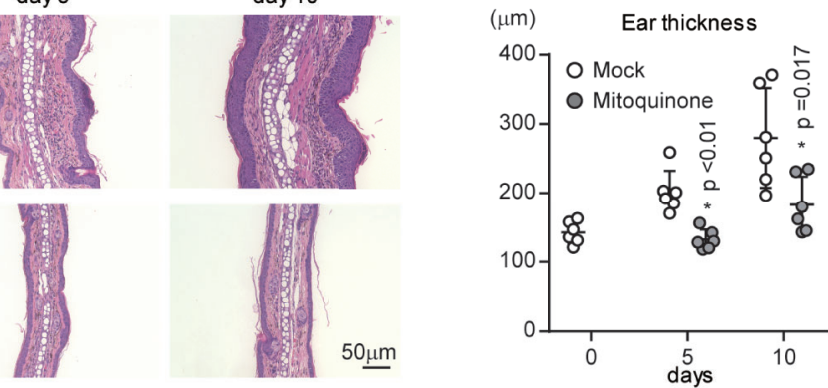

D
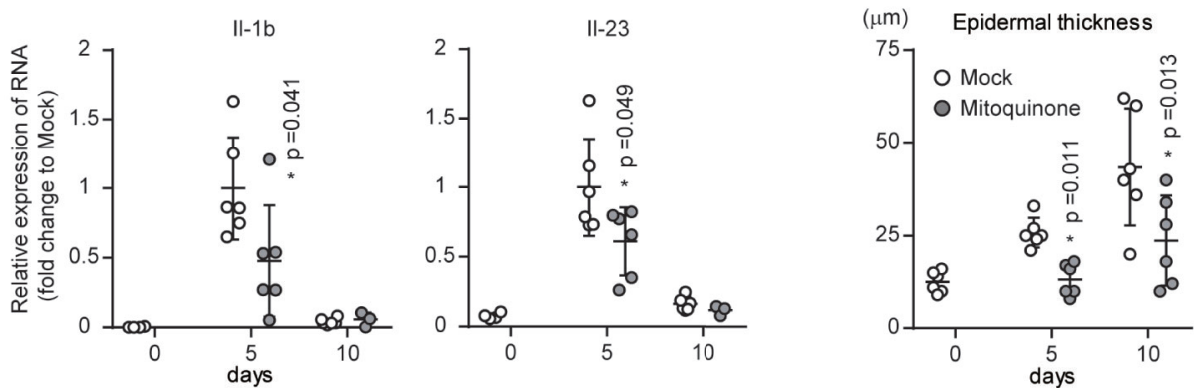

FIGURE 6 | Effects of a mitochondrial ROS inhibitor on psoriatic inflammation in vivo. WT mice were topically treated with IMQ for 9 consecutive days and received either mitoquinone or mock treatment every 2-3 days by intraperitoneal injection, beginning on day 0. (A) Cumulative Psoriasis Area Severity Index (PASI) scores were calculated based on the individual scores for redness, scaling, and stiffness daily until 9 days after IMQ application. Data are shown as means \pm SDs ( $=4-6)$. (B, C) Microscopy of cross-sections of mouse ears stained with hematoxylin and eosin (B). Changes in ear (C upper) and epidermal (C lower) thickness over time. Scale bars, $50 \mu \mathrm{m}$. (D) Real-time PCR analysis was performed to assess mRNA levels in IMQ-treated mouse ears on days 5 and 10 ( $n \geq 3$ ). The results were normalized to $18 \mathrm{~S}$ expression and are shown as means \pm SDs. ${ }^{*} p<0.05$ versus mock treatment. Data are representative of three independent experiments.

that mtROS suppression attenuates the exacerbation of psoriasis in a mouse model (Figure 6). The generation of mtROS by IMQ stimulation may promote the production of inflammatory cytokines, as well as DC maturation. Because MitoQ, an inhibitor of mtROS, also suppressed IMQ-induced DC activation in vitro (Figure 5), mtROS inhibition may serve as a therapeutic target for immune disorders associated with DC activation. Importantly, we could not analyze the roles of keratinocytes in psoriasis pathogenesis. A previous study demonstrated the critical role of mtROS from the mitochondrial 
ETC during keratinocyte differentiation (63). However, the role of mtROS in psoriasis-associated keratinocytes is unknown. Elucidation of the role of mtROS in keratinocytes may be important for the development of psoriasis treatments. Furthermore, several studies have shown that mtROS promote Th17 cell generation and exacerbation of Th17-related autoimmune disease $(64,65)$. Based on the prior results and our findings in this study, mtROS may be involved in the activation of DCs and Th17 cells in autoimmune diseases. Therefore, we anticipate that the inhibition of mtROS will be a useful therapeutic target for DC- and Th17-related autoimmune diseases.

The relationship between mitochondria and psoriasis has been unclear thus far. In this study, we have demonstrated that p32/C1qbp is an important factor for the exacerbation of psoriasis through mtROS. Because p $32 / \mathrm{C} 1 \mathrm{qbp}$ is a multifunctional chaperone protein in mitochondria (66), we suspect that $\mathrm{p} 32 / \mathrm{C} 1 \mathrm{qbp}$ is involved in other psoriasis-related pathways. Several previous studies showed that p32/C1qbp regulates mitochondrial translation and metabolism $(34,35$, 67). Because metabolites of fatty acid oxidation and the TCA cycle were decreased in p32-deficient DCs (Figure 4E), intracellular metabolism involving fatty acid oxidation and the TCA cycle may contribute to the exacerbation of psoriasis. A recent study demonstrated that intracellular metabolism of fatty acids and mtROS induced IL-23-mediated psoriatic inflammation through DC activation (30). The present study also showed that mtROS induce IL-1 $\beta$ and IL-23-mediated psoriatic inflammation. However, our study did not show a relationship between mitochondrial metabolism and psoriasis. Therefore, further research is required regarding the relationship between autoimmune diseases and mitochondrial metabolism.

In summary, we have demonstrated that the mitochondrial protein $\mathrm{p} 32 / \mathrm{Clqbp}$ has important roles in the exacerbation of psoriasis and DC activation. Recent studies in mice revealed that p32/C1qbp is involved in erythropoiesis, innate immunity, and the integrated stress response $(34,41,68)$. Moreover, p32/C1qbp transcription patterns are associated with the outcomes of influenza A, actinic keratosis, and cancers (69-71). Considering the varied expression of $\mathrm{p} 32 / \mathrm{C} 1 \mathrm{qpb}$ in many cell types and organs, we promote further study on the association between $\mathrm{p} 32 / \mathrm{Cl}$ qbp and mtROS focusing on the pathogenesis of autoimmune diseases in mice and humans. In particular, because p32/C1qbp and mtROS may have important roles in autoimmune diseases, further analyses are essential.

\section{LIMITATIONS OF THE STUDY}

There were a few limitations in this study. First, we could not fully rule out the indirect effects of epithelial cells in the mouse model. Second, we could not measure the expression of p32/ C1qbp in a group of patients with psoriasis, although past microarray data suggest that $\mathrm{p} 32 / \mathrm{Cl} \mathrm{qbp}$ is involved in the exacerbation of psoriasis (38). Third, we could not fully demonstrate mechanistic links between the p32/C1qbpmtROS-inflammasome and DC maturation. Therefore, further investigations are required to conclusively determine the functions of $\mathrm{p} 32 / \mathrm{C} 1 \mathrm{qbp}$ in psoriasis.

\section{DATA AVAILABILITY STATEMENT}

The datasets presented in this study can be found in online repositories. The names of the repository/repositories and accession number(s) can be found in the article/Supplementary Material.

\section{ETHICS STATEMENT}

The animal research protocols were approved by the Committee of Ethics on Animal Experiments, Faculty of Medical Sciences, Kyushu University.

\section{AUTHOR CONTRIBUTIONS}

KG and DK designed the study. SM, KG, YN, DS, and YT carried out the experiments. SM and KG prepared the figures and wrote the manuscript. SO and DK supervised the experiments. KG, SO, and DK reviewed the experiments. All authors contributed to the article and approved the submitted version.

\section{FUNDING}

This work was supported by JSPS KAKENHI Grant Numbers JP18K11077 and JP16K19196 (both to KG) and JP20H00530 and JP17H01550 (both to DK). This work was also supported by grants from the Takeda Science Foundation, The Shin-Nihon Foundation of Advanced Medical Research, and Charitable Trust Laboratory Medicine Research Foundation of Japan (to KG).

\section{ACKNOWLEDGMENTS}

We thank our colleagues in Dr. Kang's laboratory, Dr. Takeshi Nakahara, and Masutaka Furue for their support throughout this project. We appreciate the technical support from the Research Support Center, Graduate School of Medical Sciences, Kyushu University, and the Medical Institute of Bioregulation, Kyushu University. We thank R. Ugawa for performing the transmission electron microscopic observations. We also thank Ryan Chastain-Gross, Ph.D., from Edanz Group (https://jp.edanz. com/ac) for editing a draft of this manuscript.

\section{SUPPLEMENTARY MATERIAL}

The Supplementary Material for this article can be found online at: https://www.frontiersin.org/articles/10.3389/fimmu.2021.714897/ full\#supplementary-material 


\section{REFERENCES}

1. Christophers E. Psoriasis-Epidemiology and Clinical Spectrum. Clin Exp Dermatol (2001) 26(4):314-20. doi: 10.1046/j.1365-2230.2001.00832.x

2. Danielsen K, Olsen AO, Wilsgaard T, Furberg AS. Is the Prevalence of Psoriasis Increasing? A 30-Year Followup of a Population-Based Cohort. $\mathrm{Br} J$ Dermatol (2013) 168(6):1303-10. doi: 10.1111/bjd.12230

3. Parisi R, Symmons DPM, Griffiths CEM, Ashcroft DM, Management I. Global Epidemiology of Psoriasis: A Systematic Review of Incidence and Prevalence. J Invest Dermatol (2013) 133(2):377-85. doi: 10.1038/jid.2012.339

4. Egeberg A, Andersen YMF, Thyssen JP. Prevalence and Characteristics of Psoriasis in Denmark: Findings From the Danish Skin Cohort. BMJ Open (2019) 9(3):e028116. doi: 10.1136/bmjopen-2018-028116

5. Lowes MA, Bowcock AM, Krueger JG. Pathogenesis and Therapy of Psoriasis. Nature (2007) 445(7130):866-73. doi: 10.1038/nature05663

6. Nestle FO, Kaplan DH, Barker J. Psoriasis. N Engl J Med (2009) 361(5):496509. doi: 10.1056/NEJMra0804595

7. Nestle FO, Di Meglio P, Qin JZ, Nickoloff BJ. Skin Immune Sentinels in Health and Disease. Nat Rev Immunol (2009) 9(10):679-91. doi: 10.1038/nri2622

8. Lowes MA, Suarez-Farinas M, Krueger JG. Immunology of Psoriasis. Annu Rev Immunol (2014) 32:227-55. doi: 10.1146/annurev-immunol-032713-120225

9. Beroukhim K, Danesh MJ, Nguyen C, Austin A, Koo J, Levin E. Anti-IL-23 Phase II Data for Psoriasis: A Review. J Drugs Dermatol (2015) 14(10):1093-6.

10. Brown G, Malakouti M, Wang E, Koo JY, Levin E. Anti-IL-17 Phase II Data for Psoriasis: A Review. J Dermatolog Treat (2015) 26(1):32-6. doi: 10.3109/09546634.2013.878448

11. Greb JE, Goldminz AM, Elder JT, Lebwohl MG, Gladman DD, Wu JJ, et al. Psoriasis. Nat Rev Dis Primers (2016) 2:16082. doi: 10.1038/nrdp.2016.82

12. Ho AW, Kupper TS. T Cells and the Skin: From Protective Immunity to Inflammatory Skin Disorders. Nat Rev Immunol (2019) 19(8):490-502. doi: 10.1038/s41577-019-0162-3

13. Kamata M, Tada Y. Safety of Biologics in Psoriasis. J Dermatol (2018) 45 (3):279-86. doi: 10.1111/1346-8138.14096

14. Wang A, Bai Y. Dendritic Cells: The Driver of Psoriasis. J Dermatol (2020) 47 (2):104-13. doi: 10.1111/1346-8138.15184

15. Zaba LC, Fuentes-Duculan J, Eungdamrong NJ, Abello MV, Novitskaya I, Pierson KC, et al. Psoriasis Is Characterized by Accumulation of Immunostimulatory and Th1/Th17 Cell-Polarizing Myeloid Dendritic Cells. J Invest Dermatol (2009) 129(1):79-88. doi: 10.1038/jid.2008.194

16. Perera GK, Di Meglio P, Nestle FO. Psoriasis. Annu Rev Pathol (2012) 7:385422. doi: 10.1146/annurev-pathol-011811-132448

17. Gilliet M, Conrad C, Geiges M, Cozzio A, Thurlimann W, Burg G, et al. Psoriasis Triggered by Toll-Like Receptor 7 Agonist Imiquimod in the Presence of Dermal Plasmacytoid Dendritic Cell Precursors. Arch Dermatol (2004) 140(12):1490-5. doi: 10.1001/archderm.140.12.1490

18. Barrat FJ, Coffman RL. Development of TLR Inhibitors for the Treatment of Autoimmune Diseases. Immunol Rev (2008) 223:271-83. doi: 10.1111/j.1600065X.2008.00630.x

19. Ganguly D, Chamilos G, Lande R, Gregorio J, Meller S, Facchinetti V, et al. SelfRNA-Antimicrobial Peptide Complexes Activate Human Dendritic Cells Through TLR7 and TLR8. J Exp Med (2009) 206(9):1983-94. doi: 10.1084/jem.20090480

20. Gotoh K, Tanaka Y, Nishikimi A, Nakamura R, Yamada H, Maeda N, et al. Selective Control of Type I IFN Induction by the Rac Activator DOCK2 During TLR-Mediated Plasmacytoid Dendritic Cell Activation. J Exp Med (2010) 207(4):721-30. doi: 10.1084/jem.20091776

21. Nadeem A, Ahmad SF, El-Sherbeeny AM, Al-Harbi NO, Bakheet SA, Attia SM. Systemic Inflammation in Asocial BTBR T+ tf/J Mice Predisposes Them to Increased Psoriatic Inflammation. Prog Neuropsychopharmacol (2018) 83:8-17. doi: 10.1016/j.pnpbp.2017.12.015

22. Al-Harbi NO, Nadeem A, Ahmad SF, Bakheet SA, El-Sherbeeny AM, Ibrahim KE, et al. Therapeutic Treatment With Ibrutinib Attenuates ImiquimodInduced Psoriasis-Like Inflammation in Mice Through Downregulation of Oxidative and Inflammatory Mediators in Neutrophils and Dendritic Cells. Eur J Pharmacol (2020) 877. doi: 10.1016/j.ejphar.2020.173088

23. Nadeem A, Ahmad SF, Al-Harbi NO, El-Sherbeeny AM, Alasmari AF, Alanazi WA, et al. Bruton's Tyrosine Kinase Inhibitor Suppresses Imiquimod-Induced Psoriasis-Like Inflammation in Mice Through Regulation of IL-23/IL-17A in Innate Immune Cells. Int Immunopharmacol (2020) 80. doi: $10.1016 /$ j.intimp.2020.106215
24. Beutner KR, Tyring S. Human Papillomavirus and Human Disease. Am J Med (1997) 102(5A):9-15. doi: 10.1016/S0002-9343(97)00178-2

25. Hemmi H, Kaisho T, Takeuchi O, Sato S, Sanjo H, Hoshino K, et al. Small Anti-Viral Compounds Activate Immune Cells Via the TLR7 MyD88Dependent Signaling Pathway. Nat Immunol (2002) 3(2):196-200. doi: 10.1038/ni758

26. Patel U, Mark NM, Machler BC, Levine VJ. Imiquimod 5\% Cream Induced Psoriasis: A Case Report, Summary of the Literature and Mechanism. Br J Dermatol (2011) 164(3):670-2. doi: 10.1111/j.1365-2133.2010.10124.x

27. van der Fits L, Mourits S, Voerman JS, Kant M, Boon L, Laman JD, et al. Imiquimod-Induced Psoriasis-Like Skin Inflammation in Mice Is Mediated Via the IL-23/IL-17 Axis. J Immunol (2009) 182(9):5836-45. doi: 10.4049/ jimmunol.0802999

28. Ha HL, Wang H, Pisitkun P, Kim JC, Tassi I, Tang W, et al. IL-17 Drives Psoriatic Inflammation Via Distinct, Target Cell-Specific Mechanisms. Proc Natl Acad Sci U S A (2014) 111(33):E3422-31. doi: 10.1073/pnas.1400513111

29. Gross CJ, Mishra R, Schneider KS, Medard G, Wettmarshausen J, Dittlein DC, et al. $\mathrm{K}(+)$ Efflux-Independent NLRP3 Inflammasome Activation by Small Molecules Targeting Mitochondria. Immunity (2016) 45(4):761-73. doi: 10.1016/j.immuni.2016.08.010

30. Mogilenko DA, Haas JT, L'Homme L, Fleury S, Quemener S, Levavasseur M, et al. Metabolic and Innate Immune Cues Merge Into a Specific Inflammatory Response Via the UPR. Cell (2019) 177(5):1201-16. doi: 10.1016/ j.cell.2019.03.018

31. Krainer AR, Mayeda A, Kozak D, Binns G. Functional Expression of Cloned Human Splicing Factor Sf2 - Homology to RNA-Binding Proteins, U1-70k, and Drosophila Splicing Regulators. Cell (1991) 66(2):383-94. doi: 10.1016/ 0092-8674(91)90627-B

32. Muta T, Kang D, Kitajima S, Fujiwara T, Hamasaki N. P32 Protein, a Splicing Factor 2-Associated Protein, Is Localized in Mitochondrial Matrix and Is Functionally Important in Maintaining Oxidative Phosphorylation. J Biol Chem (1997) 272(39):24363-70. doi: 10.1074/jbc.272.39.24363

33. Yagi M, Uchiumi T, Takazaki S, Okuno B, Nomura M, Yoshida S, et al. p32/ $\mathrm{gClqR}$ Is Indispensable for Fetal Development and Mitochondrial Translation: Importance of Its RNA-Binding Ability. Nucleic Acids Res (2012) 40(19):9717-37. doi: 10.1093/nar/gks774

34. Sasaki K, Gotoh K, Miake S, Setoyama D, Yagi M, Igami K, et al. p32 is Required for Appropriate Interleukin-6 Production Upon LPS Stimulation and Protects Mice From Endotoxin Shock. EBioMedicine (2017) 20:161-72. doi: 10.1016/j.ebiom.2017.05.018

35. Gotoh K, Morisaki T, Setoyama D, Sasaki K, Yagi M, Igami K, et al. Mitochondrial p32/C1qbp Is a Critical Regulator of Dendritic Cell Metabolism and Maturation. Cell Rep (2018) 25(7):1800-15 e4. doi: 10.1016/j.celrep.2018.10.057

36. Feichtinger RG, Olahova M, Kishita Y, Garone C, Kremer LS, Yagi M, et al. Biallelic C1QBP Mutations Cause Severe Neonatal-, Childhood-, or LaterOnset Cardiomyopathy Associated With Combined Respiratory-Chain Deficiencies. Am J Hum Genet (2017) 101:525-38. doi: 10.1016/ j.ajhg.2017.08.015

37. Saito T, Uchiumi T, Yagi M, Amamoto R, Setoyama D, Matsushima Y, et al. Cardiomyocyte-Specific Loss of Mitochondrial p32/C1qbp Causes Cardiomyopathy and Activates Stress Responses. Cardiovasc Res (2017) 113:1173-85. doi: 10.1093/cvr/cvx095

38. Martel BC, Litman T, Hald A, Norsgaard H, Lovato P, Dyring-Andersen B, et al. Distinct Molecular Signatures of Mild Extrinsic and Intrinsic Atopic Dermatitis. Exp Dermatol (2016) 25(6):453-9. doi: 10.1111/exd.12967

39. Bravo A, Kavanaugh A. Bedside to Bench: Defining the Immunopathogenesis of Psoriatic Arthritis. Nat Rev Rheumatol (2019) 15(11):645-56. doi: 10.1038/ s41584-019-0285-8

40. Feichtinger RG, Sperl W, Bauer JW, Kofler B. Mitochondrial Dysfunction: A Neglected Component of Skin Diseases. Exp Dermatol (2014) 23(9):607-14. doi: 10.1111/exd.12484

41. Gotoh K, Kunisaki Y, Mizuguchi S, Setoyama D, Hosokawa K, Yao H, et al. Mitochondrial Protein Synthesis Is Essential for Terminal Differentiation of CD45(-) TER119(-)Erythroid and Lymphoid Progenitors. iScience (2020) 23 (11):101654. doi: 10.1016/j.isci.2020.101654

42. Gotoh K, Tanaka Y, Nishikimi A, Inayoshi A, Enjoji M, Takayanagi R, et al. Differential Requirement for DOCK2 in Migration of Plasmacytoid Dendritic 
Cells Versus Myeloid Dendritic Cells. Blood (2008) 111(6):2973-6. doi: 10.1182/blood-2007-09-112169

43. Kelso GF, Porteous CM, Coulter CV, Hughes G, Porteous WK, Ledgerwood EC, et al. Selective Targeting of a Redox-Active Ubiquinone to Mitochondria Within Cells: Antioxidant and Antiapoptotic Properties. J Biol Chem (2001) 276(7):4588-96. doi: 10.1074/jbc.M009093200

44. Gotoh K, Takata Y, Nakashima Y, Mizuguchi S, Komori K, Kang D. Metabolic Analysis of Mouse Bone-Marrow-Derived Dendritic Cells Using an Extracellular Flux Analyzer. STAR Protoc (2021) 2(2):100401. doi: 10.1016/ j.xpro.2021.100401

45. Ueno H, Klechevsky E, Morita R, Aspord C, Cao T, Matsui T, et al. Dendritic Cell Subsets in Health and Disease. Immunol Rev (2007) 219:118-42. doi: 10.1111/j.1600-065X.2007.00551.x

46. Kawai T, Akira S. Toll-Like Receptors and Their Crosstalk With Other Innate Receptors in Infection and Immunity. Immunity (2011) 34(5):637-50. doi: 10.1016/j.immuni.2011.05.006

47. Al-Harbi NO, Nadeem A, Ahmad SF, Alanazi MM, Aldossari AA, Alasmari F. Amelioration of Sepsis-Induced Acute Kidney Injury Through Inhibition of Inflammatory Cytokines and Oxidative Stress in Dendritic Cells and Neutrophils Respectively in Mice: Role of Spleen Tyrosine Kinase Signaling. Biochimie (2019) 158:102-10. doi: 10.1016/j.biochi.2018.12.014

48. Hansel A, Gunther C, Ingwersen J, Starke J, Schmitz M, Bachmann M, et al. Human Slan (6-Sulfo LacNAc) Dendritic Cells are Inflammatory Dermal Dendritic Cells in Psoriasis and Drive Strong TH17/TH1 T-Cell Responses. J Allergy Clin Immunol (2011) 127(3):787-94 e1-9. doi: 10.1016/j.jaci.2010.12.009

49. Li C, Bi Y, Li Y, Yang H, Yu Q, Wang J, et al. Dendritic Cell MST1 Inhibits Th17 Differentiation. Nat Commun (2017) 8:14275. doi: 10.1038/ncomms14275

50. Nadeem A, Ahmad SF, Al-Harbi NO, Ibrahim KE, Alqahtani F, Sobeai HMA, et al. Inhibition of Interleukin-2-Inducible T-Cell Kinase Causes Reduction in Imiquimod-Induced Psoriasiform Inflammation Through Reduction of Th17 Cells and Enhancement of Treg Cells in Mice. Biochimie (2020) 179:146-56. doi: 10.1016/j.biochi.2020.09.023

51. Ravindran JS, Owen P, Lagan A, Lewis J, Korendowych E, Welsh K, et al. Interleukin 1alpha, Interleukin lbeta and Interleukin 1 Receptor Gene Polymorphisms in Psoriatic Arthritis. Rheumatology (Oxford) (2004) 43 (1):22-6. doi: 10.1093/rheumatology/keg443

52. Cai Y, Xue F, Quan C, Qu M, Liu N, Zhang Y, et al. A Critical Role of the IL1beta-IL-1R Signaling Pathway in Skin Inflammation and Psoriasis Pathogenesis. J Invest Dermatol (2019) 139(1):146-56. doi: 10.1016/ j.jid.2018.07.025

53. Mansouri B, Richards L, Menter A. Treatment of Two Patients With Generalized Pustular Psoriasis With the Interleukin-1beta Inhibitor Gevokizumab. Br J Dermatol (2015) 173(1):239-41. doi: 10.1111/bjd.13614

54. Skendros P, Papagoras C, Lefaki I, Giatromanolaki A, Kotsianidis I, Speletas M, et al. Successful Response in a Case of Severe Pustular Psoriasis After Interleukin1beta Inhibition. Br J Dermatol (2017) 176(1):212-5. doi: 10.1111/bjd.14685

55. Sutton CE, Lalor SJ, Sweeney CM, Brereton CF, Lavelle EC, Mills KH. Interleukin-1 and IL-23 Induce Innate IL-17 Production From Gammadelta T Cells, Amplifying Th17 Responses and Autoimmunity. Immunity (2009) 31 (2):331-41. doi: 10.1016/j.immuni.2009.08.001

56. Ghoreschi K, Laurence A, Yang XP, Tato CM, McGeachy MJ, Konkel JE, et al. Generation of Pathogenic T(H)17 Cells in the Absence of TGF-Beta Signalling. Nature (2010) 467(7318):967-71. doi: 10.1038/nature09447

57. Cai Y, Shen X, Ding C, Qi C, Li K, Li X, et al. Pivotal Role of Dermal IL-17Producing Gammadelta T Cells in Skin Inflammation. Immunity (2011) 35 (4):596-610. doi: 10.1016/j.immuni.2011.08.001

58. Zhou Q, Mrowietz U, Rostami-Yazdi M. Oxidative Stress in the Pathogenesis of Psoriasis. Free Radic Biol Med (2009) 47(7):891-905. doi: 10.1016/ j.freeradbiomed.2009.06.033

59. Khmaladze I, Nandakumar KS, Holmdahl R. Reactive Oxygen Species in Psoriasis and Psoriasis Arthritis: Relevance to Human Disease. Int Arch Allergy Immunol (2015) 166(2):135-49. doi: 10.1159/000375401
60. Plenkowska J, Gabig-Ciminska M, Mozolewski P. Oxidative Stress as an Important Contributor to the Pathogenesis of Psoriasis. Int J Mol Sci (2020) 21 (17):6206. doi: 10.3390/ijms21176206

61. Kim HR, Lee A, Choi EJ, Hong MP, Kie JH, Lim W, et al. Reactive Oxygen Species Prevent Imiquimod-Induced Psoriatic Dermatitis Through Enhancing Regulatory T Cell Function. PloS One (2014) 9(3):e91146. doi: 10.1371/ journal.pone.0091146

62. Dan Dunn J, Alvarez LA, Zhang X, Soldati T. Reactive Oxygen Species and Mitochondria: A Nexus of Cellular Homeostasis. Redox Biol (2015) 6:472-85. doi: 10.1016/j.redox.2015.09.005

63. Hamanaka RB, Glasauer A, Hoover P, Yang S, Blatt H, Mullen AR, et al. Mitochondrial Reactive Oxygen Species Promote Epidermal Differentiation and Hair Follicle Development. Sci Signal (2013) 6(261):ra8. doi: 10.1126/ scisignal. 2003638

64. Kaufmann U, Kahlfuss S, Yang J, Ivanova E, Koralov SB, Feske S. Calcium Signaling Controls Pathogenic Th17 Cell-Mediated Inflammation by Regulating Mitochondrial Function. Cell Metab (2019) 29(5):1104-18 e6. doi: 10.1016/j.cmet.2019.01.019

65. Zhang D, Jin W, Wu R, Li J, Park SA, Tu E, et al. High Glucose Intake Exacerbates Autoimmunity Through Reactive-Oxygen-Species-Mediated TGF-Beta Cytokine Activation. Immunity (2019) 51(4):671-81 e5. doi: 10.1016/j.immuni.2019.08.001

66. Jiang J, Zhang Y, Krainer AR, Xu RM. Crystal Structure of Human P32, a Doughnut-Shaped Acidic Mitochondrial Matrix Protein. Proc Natl Acad Sci USA (1999) 96(7):3572-7. doi: 10.1073/pnas.96.7.3572

67. Fogal V, Richardson AD, Karmali PP, Scheffler IE, Smith JW, Ruoslahti E. Mitochondrial P32 Protein Is a Critical Regulator of Tumor Metabolism Via Maintenance of Oxidative Phosphorylation. Mol Cell Biol (2010) 30(6):130318. doi: 10.1128/MCB.01101-09

68. Sasaki K, Uchiumi T, Toshima T, Yagi M, Do Y, Hirai H, et al. Mitochondrial Translation Inhibition Triggers ATF4 Activation, Leading to Integrated Stress Response But Not to Mitochondrial Unfolded Protein Response. Biosci Rep (2020) 40(11):BSR20201289. doi: 10.1042/BSR20201289

69. Chatzopoulou F, Gioula G, Kioumis I, Chatzidimitriou D, Exindari M. Identification of Complement-Related Host Genetic Risk Factors Associated With Influenza A(H1N1)pdm09 Outcome: Challenges Ahead. Med Microbiol Immunol (2019) 208(5):631-40. doi: 10.1007/s00430-018-0567-9

70. Saha SK, Kim KE, Islam SMR, Cho SG, Gil M. Systematic Multiomics Analysis of Alterations in C1QBP mRNA Expression and Relevance for Clinical Outcomes in Cancers. J Clin Med (2019) 8(4):513. doi: 10.3390/jcm8040513

71. Trager MH, Rizk E, Rose S, Zhu K, Lau B, Fullerton BT, et al. Transcriptomic Analysis Identifies Differences in Gene Expression in Actinic Keratoses After Treatment With Imiquimod and Between Responders and Non Responders. Sci Rep (2021) 11(1):8775. doi: 10.1038/s41598-021-88424-z

Conflict of Interest: The authors declare that the research was conducted in the absence of any commercial or financial relationships that could be construed as a potential conflict of interest.

Publisher's Note: All claims expressed in this article are solely those of the authors and do not necessarily represent those of their affiliated organizations, or those of the publisher, the editors and the reviewers. Any product that may be evaluated in this article, or claim that may be made by its manufacturer, is not guaranteed or endorsed by the publisher.

Copyright (c) 2021 Mizuguchi, Gotoh, Nakashima, Setoyama, Takata, Ohga and Kang. This is an open-access article distributed under the terms of the Creative Commons Attribution License (CC BY). The use, distribution or reproduction in other forums is permitted, provided the original author(s) and the copyright owner(s) are credited and that the original publication in this journal is cited, in accordance with accepted academic practice. No use, distribution or reproduction is permitted which does not comply with these terms. 